\title{
Cross Layer Based Congestion Resistant Routing using Allied ARQ/FEC for Video Transmission for WSN
}

\author{
S.Lakshmi, S.Selvakumar Raja
}

\begin{abstract}
An analytical model is considered for reducing jamming in video transmission for video traffic related applications. Cross layer based congestion resistant routing using allied ARQ/FEC with LDPC scheme is proposed for reducing the error control in video streaming. Allied ARQ with FEC mechanism is applied during packet reception process. Active pass on nodes are selected for the error free transmission and cross layer mechanism is applied for choosing better routing policy. The received packets are checked for its correctness if it is corrupted then it is verified by using the Received Signal Strength Indicator (RSSI). Packets that corrupted are encoded and retransmitted by using low density parity check codes for reducing further retransmissions.
\end{abstract}

Keywords: Cross layer, Erroneous Detection, ARQ/FEC, Low density parity check

\section{INTRODUCTION}

These days, alongside the expanding mixed media applications, video traffic is blasting over remote systems in different long range social communication applications. Error controlling schemes for unicast video transmission incorporates Forward Error Correction (FEC), Automatic Repeat reQuest (ARQ), and error suppression [1]. FEC, an open-loop error control conspires that adds repetition to the bit stream in preceding transmission. It is appropriate for correspondence situations where the input from the recipient is not attainable, for example, intuitive conversational administrations. Since no feedback is accepted, packet errors should be anticipated, and repetition is intended for a scope of conceivable parcel misfortune acknowledge. Nonetheless, just one of the misfortune realizations really occurs, so FEC normally winds up being either wasteful or incapable [2]. At the point when input is possible by video streaming, ARQ plans would be more proficient than FEC [3]. With ARQ, loss of packets due to errors need not be anticipated as with FEC, yet just watched and results to the server. The repetition is then picked to precisely coordinate the loss. Cross layer design handles transmission for multimedia over wireless networks, it solves global optimisation of system performance [4]. If more than one users need to get similar information then multicast transmission is preferred and it can offer a few favourable circumstances over unicast transmission like high data transfer capacity rate and decreased resource

Revised Manuscript Received on October 25, 2019.

S.Lakshmi, Research Scholar, Department Of Ece, Scsvmv University, Kanchipuram, India

Dr.S.Selvakumar Raja, Principal, Kakatiya Institute Of Technology \&Science For Women, Nizambad, Telangana, India prerequisites at the sender and middle of the transmitting nodes. As the quantity of multicast users expands then reserved channel capacity increments. In any case, the huge number of users likewise makes error control additional challenging, on the grounds that various users experience various losses. This is particularly valid in remote multicast, where high error rates seem to be usual. Here, we consider video transmission among wireless sensor network which aims to maintain video quality that received by all users should attain a certain pre-specified level, with reducing the energy usage for video transmission. Two error control schemes allied ARQ/FEC and LDPC is considered for error free packet transmission, along with the Markov decision process algorithm.

\section{RELATED WORKS:}

Various research works have been analysed for video transmission over remote systems and to deliver better throughput at the data receiving end. Among them some related works are elaborated here. Hybrid application-layer error correction was proposed which highly consisting of FEC and retransmissions [5]. Integrated joint source-channel coding (IJSCC) structure is considered here channel coding, error resilient coding and error concealment are all being analysed for getting good video quality at the receiver. Packet loss compensation is reduced using FEC and source codes. New FEC designation calculation for unequal error protection (UEP) of video transmission over remote diverts was proposed [6]. A UEP method that takes in consideration of group of pictures (GOP) and macro blocks in a video outline, an upgraded video error proliferation model, to be specific expected number of macro blocks error proliferation (ENMEP) is additionally projected to quantify the measure of error that spreader through erroneous transmission.

An analysis of error control technique based on MARKOV process is carried out [7], here two inputs are combined such as video packets generation and channel model both are tuned by Markov states. Once the packet generation process done error control check is carried out using Markov states and the error packets are retransmitted back. To improve the video quality hybrid ARQ with selective retransmission model is considered. A control system approach to transmission distortion modelling was proposed [8], here wireless video transmission and decoding system is considered as linear system with the transmission errors as system input and the transmission distortion as output. For estimation of instant transmission distortion low-pass filtering behaviour is analysed for video encoders. It permits the encoder to foresee

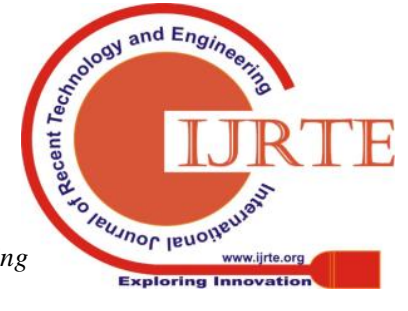


the transmission bending even before the video is compacted and transmitted since it is a predictive model. A cross-layer design for optimizing 3-D wavelet scalable video transmission [9] was proposed. This plan comprises of a large scale timescale and a smaller scale timescale rate control plans that takes place at the application layer and the system sub-layer individually. The large scale rate control utilizes transmission capacity estimation to accomplish ideal piece designation with reduced distortion. The small scale rate control utilizes a versatile mapping of packets from video orders to proper system to amplify the transmission assurance to the significant video packets.

A scheduling framework dubbed delAy Stringent COded Transmission (ASCOT) [10] was proposed for the purpose of multi-homed correspondence with delay-constrained High Definition (HD) video in heterogeneous remote systems. The impairments like feedback channel status and input video data was taken and by adapting FEC, coding redundancy and data allocation to achieve target quality. Dynamic programming approach was proposed [11] to decide the ideal slicing and UEP arrangement for every video outline in a down to earth way that is agreeable with the AVC/H.264 standard. Likewise new rate and distortion estimation strategies were proposed at the encoder side so as to proficiently assess the target work for a slice configuration. For rate distortion optimal video delivery, a classical independent-layer transport model for optimal slicing, a FEC coding is performed at the link layer. However, the application layer controls the FEC code rate with the constraint that a given IP packet is subject to constant channel protection. To support quality of service with light weight error concealment a joint framework [12] for video transmission was proposed. Error Concealment scheme assumes an indispensable job to improve Quality of Experience (QoE) by keeping up a satisfactory quality at the accepting finishes. The fundamental destinations of the proposed system are to boost the system throughput and to conceal the impacts created by dropped video packets. To control the information rate, Scalable High effectiveness Video Coding (SHVC) is applied at sight and sound sensor hubs with variable Quantization Parameters (QPs). Stream control transmission convention (SCTP) [13] is a significant transport layer solution for executing simultaneous multipath move over heterogeneous remote systems with multi homed terminals. To convey the energy productive and good Put interactive media gushing over different remote systems, this paper introduces an Energy and good Put Optimized concurrent path (EPOC) arrangement. A joint forward error correction coding and rate distribution method was projected to limit energy utilization while fulfilling good Put requirement. Cross-layer fairness driven (CL/FD) SCTP-based concurrent multipath arrangement (CMA-CL/FD) [14] was proposed to improve video conveyance execution, while staying reasonable for the contending TCP streams. CMA-CL/FD uses a cross-layer way to deal with screen and investigate way quality, which incorporates remote channel estimations at the information connection layer and rate/BW estimation. Besides, an imaginative window-based component is applied for stream control to adjust conveyance reasonableness and proficiency. Error Concealment aware encoding [15] scheme was presented to improve decoded video feature in situations subject to transmission errors and information errors. This scheme relies on adaptable coding approach where good EC techniques to be utilized at the decoder are ideally determined at the encoder and motioned to the decoder through supplemental improvement data messages. 3-D engrossing Markov chain model [16] is introduced to precisely compute the parcel transmission time when both the FEC and ARQ instruments are utilized. FEC/ARQ mechanisms are used at the data-link layer to avoid and recover the channel errors.

\section{PROPOSED METHOD:}

Pass on-node Selection and corruption attentive process with cross layer technique is proposed here. Cross layer based congestion resistant routing Error control (CLCRR-EC) with Allied ARQ/FEC is proposed. Allied ARQ/FEC is applied for corruption control retransmission process. The number of corrupted bytes in the received packets is identified using RSSI time series for safety measures. The hand-on node selection should be done under sensible operating conditions like Channel Estimation Errors (CEEs) on all wireless channels; self-regulating and intrusion power constraint. Erroneous packet detection algorithm finds out the number of debased or erroneous bytes in a received packet. In order to discover falsification, a high-resolution RSSI sampling procedure is applied during reception of packet and retransmits the data again using LDPC. An optimal pass on nodes with minimum selection overhead can be identified by the power of nodes outstanding ability. Besides, relaying is done only when the sink node is insufficient for decoding the data that is transmitted by the source. To overcome the packet failure, efficient pass on node selection is presented. Allied ARQ/FEC with hand-on node selection is deployed for transmitting the data's successfully over intermediates from initial node to destination that parallel improves the throughput level by reducing retransmission count.

\section{SUPPLEMENT NODE SELECTION:}

If the packet transmission between the path $\mathrm{P}$ (source hub) to Qi and Qi to R gets fails, then new supplement node 'Qj' is elected. Adaptive supplement node 'Qi' selection process includes passing control messages between source and supplement nodes. S broadcasts R_req to its neighbours and the supplement node with high channel bandwidth sent $\mathrm{R}$ _rep back to the $\mathrm{S}$ node. If path connecting 'S' to 'SPi' and 'Qi' to ' $R$ ' fails to forward the sensed packets, then ' $P$ ' broadcasts again R_req and retransmits the failed message. This supplement node selection provides the complete transmission of INFORMATION message. Markov process is applied for identifying the supplement node state.

Tx: Node ' $\mathrm{P}$ ' sends new data. 'Supplement relay' is considered as node.

Qi: If current SPi node receives data from source 'S', then it passes the information to the node ' $\mathrm{D}$ ', but the path gets failed.

QTx: In state transition process, the state might take two fractions: type

i. First form of QTx state: Same information is retransmitted by source $\mathrm{P}$ itself by selecting another supplement ' $\mathrm{Qj}$ '.

ii. Second form of QTx state: Information is sent directly from ' $\mathrm{P}$ ' to ' $\mathrm{R}$ ' without selection of route. This state represents the move .

When source transfers data to ' $\mathrm{D}$ ' and supplement node is elected 
successfully as the convention state then adjustments made to the relating Tx state ( ).

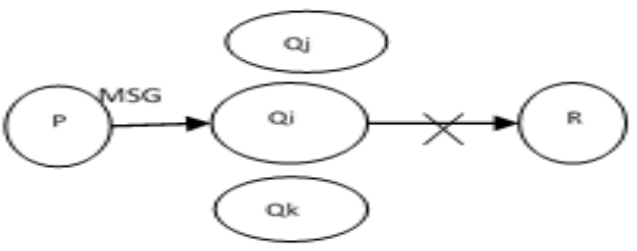

Fig.1: Actual Supplement Failure 'Qi'

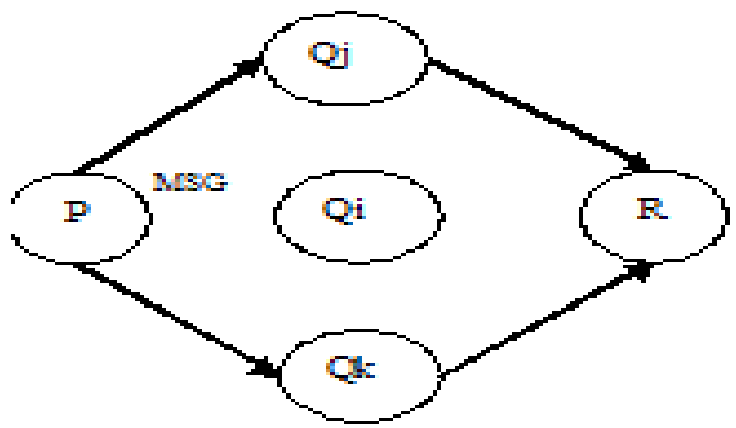

Fig.2: New Supplement Selection
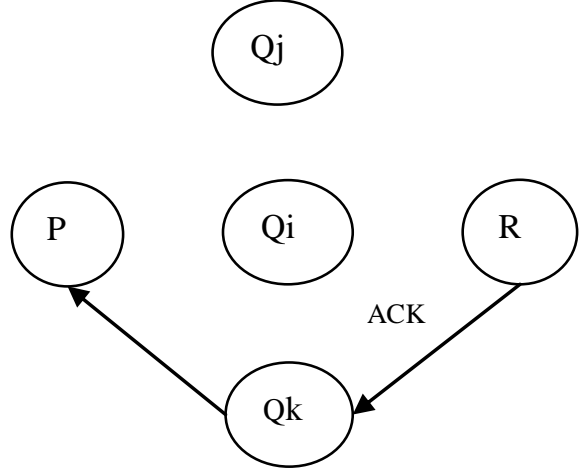

Fig.3: ACK from ' $R$ '
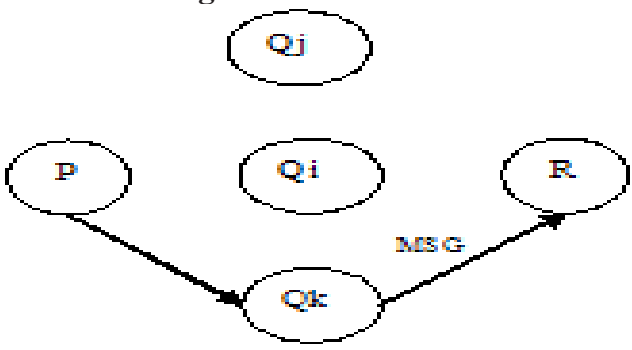

Fig.4: Retransmission

$\mathrm{RS}$ : Information is passed to ' $\mathrm{R}$ ' once the supplement node is selected and it goes to the separate state from RS. When supplement selection is incapable for processing then protocol gives back the RTx state. Now ' $\mathrm{P}$ ' retransmits data and commences new election of supplement node. Protocol transitions of states defined in Equation 2.

$$
u^{(b)} T x=\left\{\begin{aligned}
\text { for } u^{(a)}=T x, c_{P R}^{(a)}=G, \\
\text { or } u^{(a)}=R, c_{Q R}^{(a)}=G \\
\text { or } u^{(a)}=A S, c_{P R}^{(a)}, c_{P Q}^{(a)}, c_{Q R}^{(a)}=G, \\
\sum_{Q=1}^{Q=-1} I_{G}\left(c_{P Q}^{(a)}\right) I_{G}\left(c_{Q R}^{(a)}\right)=0
\end{aligned}\right.
$$

For duplicating the allied ARQ/FEC protocol, only P- R, P- Q and $\mathrm{Q}-\mathrm{R}$ radio channels are required. The tuple set $\mathrm{D}$ has all appropriate arrangements for the quad-ruple $d_{a}=\left(u^{(a)}, c_{P R}^{(a)}, c_{P Q}^{(a)}, c_{Q R}^{(a)}\right)$. There are $|D|=16$ novel tuples that consists of all conceivable states move in the structure. The utility $\boldsymbol{u}^{(b)}=f\left(\boldsymbol{d}_{a}\right)_{\text {explaining rule state transitions of }}$ allied ARQ/FEC with an adaptive intermediate supplement that can be defined as in equations 1,2 and 3 .

$$
u^{(b)} Q=\left\{\begin{aligned}
& \operatorname{for} u^{(a)}= T x, c_{P R}^{(a)}=B, c_{P Q}^{(a)}=G, \\
& \operatorname{or}^{(a)}=A S, c_{P R}^{(a)}=B, c_{P Q}^{(a)}, c_{Q R}^{(a)}=G, \\
& \sum_{Q=1}^{Q-1} I_{G}\left(c_{P Q}^{(a)}\right) I_{G}\left(c_{Q R}^{(a)}\right)=0
\end{aligned}\right.
$$

$$
u^{(b)} A S=\left\{\begin{aligned}
& \text { for } \quad u^{(a)}=T x, c_{P R}^{(a)}=B, c_{P Q}^{(a)}=B \\
& \text { or } u^{(a)}=Q, c_{Q R}^{(a)}=B \\
& \text { or } u^{(a)}=R S, c_{P R}^{(a)}=G, \\
& \sum_{Q=1}^{N} I_{G}\left(c_{P Q}^{(a)}\right) I_{G}\left(c_{Q R}^{(a)}\right)=0
\end{aligned}\right.
$$

Based on the state transition probability matrix $\mathrm{P}$, state transition or switching probabilities from tuple $d_{a}$ to $d_{b}$ can be achieved and it comprises a number of switching probabilities among tuples. Switching possibilities is computed by equation 4 .

$$
P_{a b}=\left\{\begin{array}{l}
\operatorname{Ps}\left(c_{P R}^{(b)} \mid c_{P R}^{(a)}\right) \prod_{Q=Q i}^{Q n} \operatorname{Ps}\left(c_{P Q}^{(b)} \mid c_{P Q}^{(a)}\right) \operatorname{Ps}\left(c_{Q R}^{(b)} \mid c_{Q R}^{(a)}\right) \\
\quad \text { for } u^{(b)}=f\left(d_{a}\right) \\
0 ; \quad \text { otherwise }
\end{array}\right.
$$

If a packet is sent effectively to the node dest ' $R$ ' then the ARQ/FEC hybrid protocol returns to initial Tx state and gets ready for next set of transmission. The achievement rate $Y_{a b}(a, b \in\{1,2, \ldots ., 16\})$ is allocated as in equation 5.

$$
Y_{a b}= \begin{cases}1 ; & \text { for } u^{(a b)}=T x, c_{P R}^{(a b)}=G \\ & \text { or } u^{(a b)}=Q, c_{Q R}^{(a b)}=G \\ 0 ; & \text { otherwise }\end{cases}
$$

Message transmission period of single packet for each transition of state and its holding time is similar and it is normalized to ' 1 '. The yield is derived by equation 6 . 


$$
\eta=\sum_{a=1}^{16} \pi_{a} \cdot \sum_{b=1}^{16} P_{a b} Y_{a b}
$$

Throughput achievement rate $Y_{a b}=1$ is achieved only when (a) the node P-R channel is superior; (b) the node P-R channel is awful and both the path $\mathrm{P}-\mathrm{Q}$ and node $\mathrm{Q}-\mathrm{R}$ channels are fine. Each time a protocol state transition $R \rightarrow T x_{\text {takes place, achievement rate }} Y_{a b}$ is owed as 1, which makes up half of all transitions. The energy consumed $\mathrm{E}_{\mathrm{ab}}$ during the time of state transition $\boldsymbol{d}_{a} \rightarrow \boldsymbol{d}_{b}$ is given in equation 7.

$$
E_{a b}=\left\{\begin{array}{lc}
E_{T x}+E_{R x}\left(I_{G}\left(c_{P R}^{(a)}\right)+\sum_{Q=1}^{N} I_{G}\left(c_{P Q}^{(a)}\right)\right) & \text { for }^{(a)} \in\{T x, A S\}, \\
\boldsymbol{E}_{T x}^{(b)} \in\{T x, Q\}, \\
\boldsymbol{I}_{G}\left(c_{Q R}^{(a)}\right) E_{R x} & \text { for } u^{(a)}=Q, \\
E_{T x}+I_{G}\left(c_{P R}^{(a)}\right) E_{R x} & \text { for } u^{(a)}=u^{(b)}=A S, \\
0 & \text { otherwise }
\end{array}\right.
$$

Holding time for the selection of adaptive supplement (AS) node is computed in equation 8 .

$$
H_{a b}=\left\{\begin{array}{l}
1+\omega \text { for } u^{(a)}=A S, \\
1 \quad \text { otherwise }
\end{array}\right.
$$

Based on the flow of radio channels, effective and energy adaptive supplement node selection is done using time-associated channels.

\section{ERRONEOUS DETECTION PROCESS:}

This error detection algorithm relies on finding precisely the number of erroneous bytes in a received packet. RSSI time series of sampling mechanism is considered as input when the receiver receives the packet. Based on the number of bit errors the corruption level is identified which is different to be the fraction of error bits in a packet. Initially the number of error bit is set to 0 and the packet corruption level is set as default value of 1 . To differentiate non-meddle associations from meddle associations, the scope of RSSI time series of examining is assessed. If current connection is a non-meddled interface, the calculation gives back the default packet corruption level of 1, i.e., the erroneous level is not discovered for non-meddled channel links and will retransmit a self-decidable parity bit whenever required. Else the calculation determines the quantity of erroneous bits created by impedance or channel interference.

Erroneous bit detected based on RSSI value and if it is lower than the assigned referencing value, then it is confirmed that the received packet bits are correct. In case the RSSI value is higher than the referencing value, then there exists interference and the packets get corrupted. Once the erroneous packet is identified, NACK is sent by the receiver to the sender, and the data corruption is computed. Sender uses adaptive encoding method for retransmitting parity packet that depends on received corruption level.

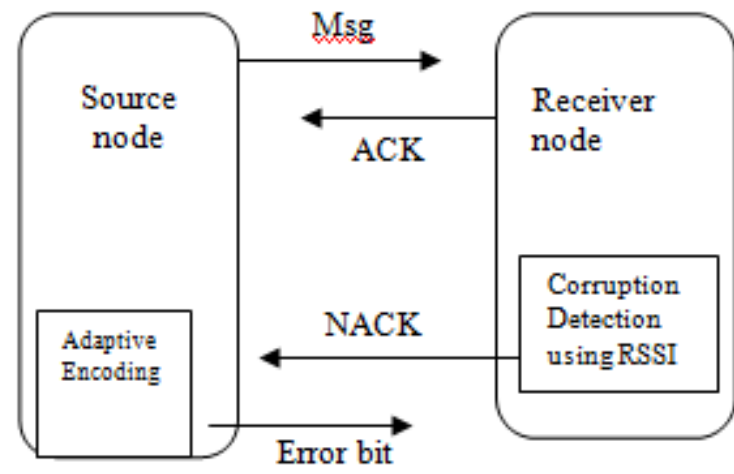

Figure: 5. Erroneous Packet Detection

Parity check $(\mathrm{P})$ is used to correct the received packet errors. Then the resultant code word is sent to the receiver. Receiving node decodes the received code word using the decoding algorithm. The decoding process recovers original information. In the decoder, LDPC decodes the message utilizing primary probabilities, disorder check and iterative decoding. Utilizing LDPC, encoding and decoding of ruined or erroneous information is conceivable to diminish the complexity nature during calculation of packets. Thereby congestion resistant routing protocol is built using allied error control mechanism ARQ/FEC with LDPC.

\section{RESULTS AND DISCUSSION:}

Network Simulator-2.35 is the simulation tool used to detect the performance of the proposed scheme CLCRR-EC. Nodes are deployed randomly in the network. MAC layer with data rate $10 \mathrm{Mbps}$ is applied. The residual parameters are presented in table 1. The proposed scheme efficiency is validated by taking base protocols ECAER and MFECT protocols.

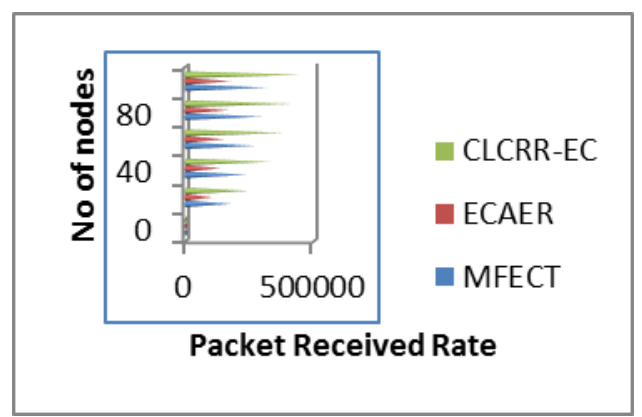

Fig.6: PRR vs No of nodes

Table 1: Simulation Parameters

\begin{tabular}{|c|c|}
\hline Parameter & Value \\
\hline Simulation Area & $1000 \times 1000 \mathrm{~m}$ \\
\hline Number of Nodes & 100 \\
\hline Channel & WirelessPhy \\
\hline MAC & 802.15 .4 \\
\hline Radio Propagation Model & Two Ray-Ground Type \\
\hline Antenna Type & Omni Directional \\
\hline CBR Interval & $1.0 \mathrm{~ms}$ \\
\hline Simulation Time & $50 \mathrm{sec}$ \\
\hline
\end{tabular}


PACKET RECEIVED RATE: Packet Received Rate (PRR) is the rate of number of packets received by the destination to the number of packets sent by the sender. It is measured by using the equation 9 ,

$$
P R R=\frac{\sum_{0}^{n} \text { packets Received }}{\sum_{0}^{n} \text { packets sent }}
$$

The figure 6 shows that the proposed scheme CLCRR-EC has higher delivery rates of packets at the receiving node compared to the existing protocols.

VIDEO QUALITY: Signal to noise ratio is estimated for obtaining the level of video quality and the processed video sequences. The proposed method CLCRR-EC gives video quality range of $31.9 \mathrm{~dB}$ which is comparatively better than the other schemes such as MFECT and ECAER and it is shown in the figure 7 . The quality of the achieved video is dependable on the error control applied during packet encoding.

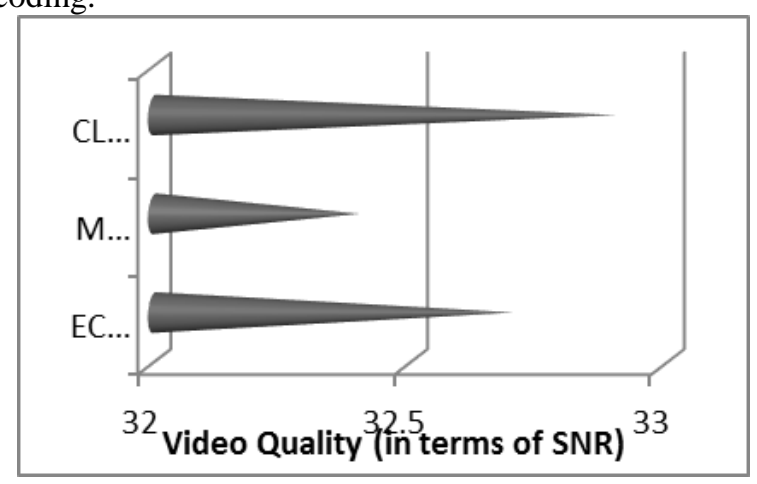

Fig.7: Peek-SNR

PACKET ERROR RATE: Scheduling the data packets reduces the loss during data transmission. By sending RSSI sampling based packet series the error rate can be reduced also the data routing cost with respect to the number of nodes participates during routing for the proposed method reduces. The error or loss rate for the method CLCRR-EC is better than the other conventional MFECT and ECAER algorithms. The fig 8 depicts packet error rates for all proposed and conventional protocols.

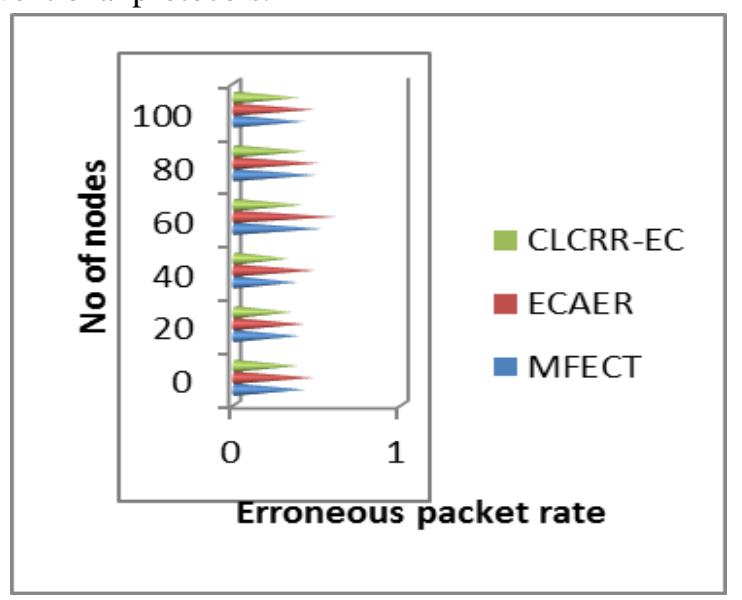

Fig.8: Packet Error Rate vs nodes

ENERGY CONSUMPTION VS NODES: The relative energy is computed for the nodes that participate in data transmission process. The energy consumed by the nodes for transferring the packets is calculated by the nodes energy drain rate. Figure 9 shows the energy consumption rate for the proposed scheme CLCRR-EC and the conventional schemes MFECT and ECAER. The proposed protocol consumes less energy for transferring the frames or video sequences.

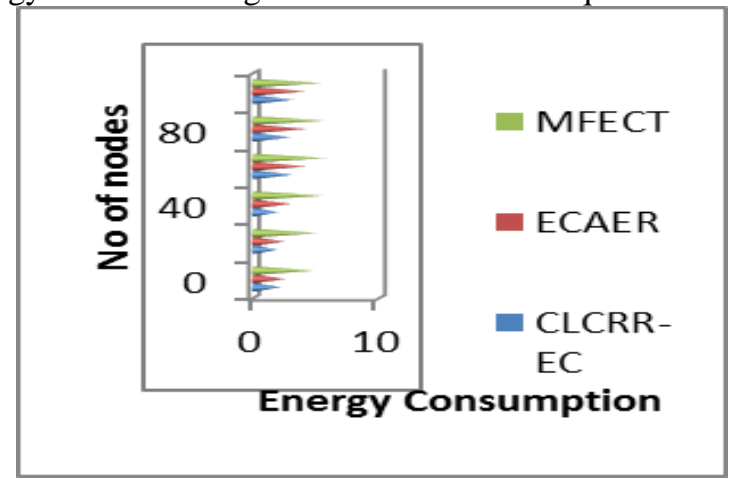

Fig.9: Energy Consumption

\section{VII.CONCLUSION:}

Cross layer based congestion resistant routing using allied ARQ/FEC with LDPC scheme is proposed significantly for reducing the error control in video applications. Allied ARQ/FEC mechanism is applied for controlling the errors during packet retransmitting process. Cross layer mechanism is applied for choosing better routing policy and error free transmissions. The received packets are checked for its correctness if it is corrupted then it is verified by using the Received Signal Strength Indicator (RSSI). The corrupted packet is encoded before retransmission using LDPC for reducing further retransmissions. This way congestion resistant routing protocol is developed and the simulation results shows better efficiency in terms of packet received rate and video quality.

\section{REFERENCES:}

1. Badia, Leonardo, Nicola Baldo, Marco Levorato, and Michele Zorzi. "A Markov Framework for error control techniques based on selective retransmission in video Transmission over wireless channels." IEEE Journal on Selected Areas in Communications 28, no. 3 (2010): 488-500.

2. 2. I. V. Bajic', "Efficient error control for wireless video multicast," in Proc. IEEE Workshop on Multimedia Signal Processing (MMSP'06), Victoria, BC, Canada, Oct. 2006, pp. 306-309.

3. Carreira, João FM, Pedro A. Assunção, Sérgio MM de Faria, Erhan Ekmekcioglu, and Ahmet Kondoz. "Error Concealment-Aware Encoding for Robust Video Transmission." IEEE, Transactions on Broadcasting (2018).

4. 4.Chang, Yoong Choon, Sze Wei Lee, and Ryoichi Komyia. "A fast forward error correction allocation algorithm for unequal error protection of video transmission over wireless channels." IEEE Transactions on Consumer Electronics 54, no. 3 (2008): 1066-1073.

5. 5. Foh, Chuan Heng, Yu Zhang, Zefeng Ni, Jianfei Cai, and King Ngi Ngan. "Optimized cross-layer design for scalable video transmission over the IEEE 802.11e networks." IEEE Transactions on Circuits and Systems for Video Technology 17, no. 12 (2007): 1665-1678.

6. 6. Harmanci, Oztan, and A. Murat Tekalp. "Rate-distortion optimal video transport Over IP allowing packets with bit errors." IEEE transactions on image processing 16, no. 5 (2007): 1315-1326.

7. 7. He, Zhihai, and Hongkai Xiong. "Transmission distortion analysis for real-time video encoding and streaming over wireless networks." IEEE transactions on Circuits and Systems for Video Technology 16, no. 9 (2006): 1051-1062. 
8. 8. Moid, Azfar, and Abraham O. Fapojuwo. "Three-dimensional absorbing Markov chain model for video streaming over IEEE 802.11 wireless networks." IEEE Transactions on Consumer Electronics 54, no. 4 (2008): 1672-1680.

9. 9. Usman, Muhammad, Ning Yang, Mian Ahmad Jan, Xiangjian He, Min Xu, and Kin-Man Lam. "A joint framework for QoS and QoE for video transmission over wireless multimedia sensor networks." IEEE Transactions on Mobile Computing 17, no. 4 (2017): 746-759.

10. 10. Van Der Schaar, Mihaela, Santhana Krishnamachari, Sunghyun Choi, and

11. Xiaofeng $\mathrm{Xu}$. "Adaptive cross-layer protection strategies for robust scalable video transmission over 802.11 WLANs." IEEE Journal on selected areas in communications 21, no. 10 (2003): 1752-1763.

12. 11. Wu, Jiyan, Chau Yuen, Ngai-Man Cheung, and Junliang Chen. "Delay- Constrained high definition video transmission in heterogeneous wireless networks with multi-homed terminals." IEEE Transactions on Mobile Computing 15, no. 3 (2015): 641-655.

13. 12. Wu, Jiyan, Bo Cheng, Ming Wang, and Junliang Chen. "Energy-aware concurrent multipath transfer for real-time video streaming over heterogeneous wireless networks." IEEE Transactions on Circuits and Systems for Video Technology 28, no. 8 (2018): 2007-2023.

14. 13. Y.Wang, J. Ostermann, and Y.-Q. Zhang, Video Processing and Communications. Upper Saddle River, NJ: Prentice-Hall, 2002.

15. 14. W. Wei and A. Zakhor, "Interference aware multipath selection for video streaming in wireless ad hoc networks," IEEE Trans. Circuits Syst. Video Technol., vol. 19,no. 2, pp. 165-178, Feb. 2009.

16. 15. Xu, Changqiao, Zhuofeng Li, Jinglin Li, Hongke Zhang, and Gabriel-Miro Muntean.Cross-layer fairness-driven concurrent multipath video delivery over heterogeneous wireless networks." IEEE Transactions on Circuits and Systems for Video Technology 25, no. 7 (2014): 1175-1189.

17. 16. Zhai, Fan, Yiftach Eisenberg, Thrasyvoulos N. Pappas, Randall Berry, and Aggelos K. Katsaggelos. "Rate-distortion optimized hybrid error control for real-time packetized video transmission." IEEE Transactions on Image Processing 15, no. 1 (2005): 40-53. 